\begin{tabular}{|c|l|}
\hline Title & V arious oscillation patterns in phase models with locally attractive and globally repul sive couplings \\
\hline Author(s) & Sato, Katsuhiko; Shima, Shin-ichiro \\
\hline Citation & $\begin{array}{l}\text { Physical review E, 92(4), 042922-1-042922-13 } \\
\text { https://doi.org/L0.1103/PhysRevE.92.042922 }\end{array}$ \\
\hline Issue Date & 2015-10-28 \\
\hline Doc URL & http://hdl.handle.net/2115/60326 \\
\hline Rights & @2015A American Physical Society \\
\hline Type & article \\
\hline File Information & PhysRevE.92.042922.pdf \\
\hline
\end{tabular}

Instructions for use 


\title{
Various oscillation patterns in phase models with locally attractive and globally repulsive couplings
}

\author{
Katsuhiko Sato ${ }^{1, *}$ and Shin-ichiro Shima ${ }^{2}$ \\ ${ }^{1}$ Research Institute for Electronic Science, Hokkaido University, Sapporo 001-0020, Japan \\ ${ }^{2}$ Graduate School of Simulation Studies, University of Hyogo, Kobe 650-0047, Japan
}

(Received 16 July 2015; published 28 October 2015)

\begin{abstract}
We investigate a phase model that includes both locally attractive and globally repulsive coupling in one dimension. This model exhibits nontrivial spatiotemporal patterns that have not been observed in systems that contain only local or global coupling. Depending on the relative strengths of the local and global coupling and on the form of global coupling, the system can show a spatially uniform state (in-phase synchronization), a monotonically increasing state (traveling wave), and three types of oscillations of relative phase difference. One of the oscillations of relative phase difference has the characteristic of being locally unstable but globally attractive. That is, any small perturbation to the periodic orbit in phase space destroys its periodic motion, but after a long time the system returns to the original periodic orbit. This behavior is closely related to the emergence of saddle two-cluster states for global coupling only, which are connected to each other by attractive heteroclinic orbits. The mechanism of occurrence of this type of oscillation is discussed.
\end{abstract}

DOI: 10.1103/PhysRevE.92.042922

PACS number(s): 05.45.Xt, 02.60.Nm

\section{INTRODUCTION}

In nature there are many systems that exhibit oscillations. Various oscillation patterns are found, depending on the type of interactions between the oscillatory elements. Recently, one of the authors and his coworkers have investigated spontaneous oscillations appearing in muscle [1-3], in which the basic units of muscle, known as sarcomeres, autonomously oscillate in length by repeated relaxation and contraction under constant conditions. Various oscillation patterns, such as traveling waves and their disrupted forms, appear in myofibrils, which are linearly connected structures comprised of sarcomeres. We constructed a mathematical model based on the structure of muscle and reproduced the oscillation patterns appearing in the myofibrils. Furthermore, we reduced the mathematical model to a phase model using the standard method $[4,5]$ and revealed that the essence of the oscillation patterns lies in characteristic interactions between sarcomeres, i.e., locally attractive but globally repulsive interactions [3].

One of the main messages from our study of oscillations in muscle is that if the elements comprising the system oscillate in length due to their intrinsic forces, e.g., active forces, and if they are mechanically connected with their adjacent elements, then the system tends to exhibit global coupling because the effect of the change in length of some element is immediately transmitted to the other elements through mechanical forces. In addition, if the whole system is confined within some region, cases of which frequently arise, then the total length of the system is approximately conserved and hence the global coupling becomes repulsive [6]. Since each element in the system is, in general, interacting with its adjacent elements, and since its interactions are attractive in many cases, oscillators induced by the active force (which are mechanically connected with their neighbors), in general, form an oscillator system in which interactions between the elements are locally attractive and globally repulsive. Here we refer to this type of system as active force oscillators.

*katsuhiko_sato@es.hokudai.ac.jp
There are many examples of active force oscillators in biological systems. For example, pulsed contraction is observed at the apical surfaces of epithelial sheets [7], in which each cell bears a contraction force at the boundaries between cells. These mechanical forces arise from the actomyosin meshwork beneath plasma membranes and are transmitted via lateral junctions between cells. Another example is shuttle streaming in the true slime mold [8], in which forces are generated by actomyosin filaments near the outer gel surrounding the ameba and are transmitted via pressure in cytosols.

To understand the behavior of active force oscillators, it is beneficial to gain knowledge of the behavior of locally-globally coupled oscillators for the reasons mentioned above. Although some investigations have been performed on locally-globally coupled oscillators $[9,10]$ that are motivated by chemical oscillators, these studies are not fully comprehensive.

In the present paper we investigate oscillators that are coupled in one dimension in locally attractive and globally repulsive fashion using a phase model that describes the dynamics of their phases. We show that this phase model exhibits various spatiotemporal patterns of the relative phase differences. These include a spatially uniform state, which corresponds to the in-phase state in the original oscillator system, a monotonically increasing state, which corresponds to traveling waves in the original system, and three types of oscillations of relative phase difference. The reason that this system can show various spatiotemporal patterns of relative phase difference is that antagonistic interactions are active, i.e., interactions that are locally attractive and globally repulsive. Depending on the relative strengths of the local and global interactions and the form of the global coupling, the system can exist in various states.

\section{BASIC EQUATIONS}

Our starting point is the phase equations that describe the dynamics of the phases of $N$ identical oscillators that interact with each other locally and globally. The form of 
these equations is given by

$$
\begin{aligned}
\frac{d \phi_{i}}{d t}= & \hat{\omega}_{0}+\hat{k}\left(B\left(\phi_{i}-\phi_{i+1}\right)+B\left(\phi_{i}-\phi_{i-1}\right)\right) \\
& +\hat{K} \sum_{j=1}^{N} A\left(\phi_{i}-\phi_{j}\right)
\end{aligned}
$$

where $\phi_{i}$ is the phase of the $i$ th oscillator $(i=1,2, \ldots, N)$, and $\hat{\omega}_{0}$ is the natural frequency of that oscillator, which is the same for all oscillators. $A$ and $B$ are $2 \pi$-periodic functions that represent global and local couplings between the oscillators, respectively. We assume that $B$ is attractive and $A$ is repulsive with respect to small phase differences, i.e., $B^{\prime}(0)<0$ and $A^{\prime}(0)>0$. The coefficients $\hat{k}$ and $\hat{K}$ are positive constants that represent the strengths of local and global interactions, respectively. The boundary conditions of Eq. (1) are taken to be the Neumann conditions, i.e., $\phi_{0}=\phi_{1}$ and $\phi_{N+1}=\phi_{N}$.

Next, we take the continuous limit of Eq. (1) by introducing new variables $x=i / N, \phi(x, t)=\phi_{i}(t)-\hat{\omega}_{0} t, \tilde{k}=\hat{k} / N^{2}$, and $\tilde{K}=\hat{K} N$ and letting $N \rightarrow \infty$. In this process the first term in Eq. (1) becomes a collection of spatial derivatives of $\phi$ and the second term becomes an integral term. If we retain only the leading terms of the $x$ derivatives of $\phi$ with respect to local couplings, we have

$$
\begin{aligned}
\frac{\partial \phi(x, t)}{\partial t}= & \tilde{k}\left(-B^{\prime}(0) \frac{\partial^{2} \phi}{\partial x^{2}}+B^{\prime \prime}(0)\left(\frac{\partial \phi}{\partial x}\right)^{2}\right) \\
& +\tilde{K} \int_{0}^{1} A\left(\phi(x, t)-\phi\left(x^{\prime}, t\right)\right) d x^{\prime}
\end{aligned}
$$

In this article we assume that $B$ is given as $B(\phi)=-B_{0} \sin \phi$ with a positive constant $B_{0}$, and hence we can set $B^{\prime \prime}(0)=0$ in the subsequent analysis. By rescaling time such that $1 / \tilde{K}$ is the unit of time and substituting $k=B_{0} \tilde{k} / \tilde{K}$ in Eq. (2), we obtain

$$
\frac{\partial \phi(x, t)}{\partial t}=k \frac{\partial^{2} \phi}{\partial x^{2}}+\int_{0}^{1} A\left(\phi(x, t)-\phi\left(x^{\prime}, t\right)\right) d x^{\prime} .
$$

This is the equation that we investigate in this article. The range of $x$ is $[0,1]$. The Neumann boundary conditions are expressed as

$$
\partial \phi(x, t) /\left.\partial x\right|_{x=0,1}=0 .
$$

We specify $A$ such that it takes the simplest possible form while enabling nontrivial behavior when globally coupled oscillations are considered. Such a form of $A$ has previously been investigated [11] and is given by

$$
A(\phi)=\sin (\phi+\alpha)+r \sin 2 \phi,
$$

where $r$ is a constant within the range $0<r<1 / 2$. $A$ is repulsive when $-\arccos (-2 r)<\alpha<\arccos (-2 r)$. From the symmetry of $A$, it is sufficient to investigate only the range $0<$ $\alpha<\arccos (-2 r)$. In this way, the control parameters of this system are $k, r$, and $\alpha$. For $k \rightarrow \infty$, Eq. (3) represents a system with local coupling only, while for $k \rightarrow+0$ it represents a system with global coupling only.

\section{A. Analytical rearrangement of Eq. (3)}

To investigate the spatiotemporal patterns of $\phi$ that obey Eq. (3), we first split $\phi$ into two components: its mean value, defined by $\Phi(t)=\int_{0}^{1} \phi\left(x^{\prime}, t\right) d x^{\prime}$; and the remaining part, defined as

$$
\hat{\phi}(x, t)=\phi(x, t)-\Phi(t)
$$

where $\hat{\phi}$ satisfies

$$
\int_{0}^{1} \hat{\phi}\left(x^{\prime}, t\right) d x^{\prime}=0
$$

for any $t$. We hereafter refer to $\hat{\phi}$ as the relative phase difference. By substituting Eq. (6) into Eq. (3) and integrating both sides with respect to $x$ from 0 to 1 , we obtain the time evolution equations for $\Phi$ and $\hat{\phi}$ as

$$
\begin{aligned}
\frac{d \Phi}{d t} & =\int_{0}^{1} d x \int_{0}^{1} d x^{\prime} A\left(\hat{\phi}(x, t)-\hat{\phi}\left(x^{\prime}, t\right)\right), \\
\frac{\partial \hat{\phi}}{\partial t}= & k \frac{\partial^{2} \hat{\phi}}{\partial x^{2}}+\int_{0}^{1} A\left(\hat{\phi}(x, t)-\hat{\phi}\left(x^{\prime}, t\right)\right) d x^{\prime} \\
& -\int_{0}^{1} d x \int_{0}^{1} d x^{\prime} A\left(\hat{\phi}(x, t)-\hat{\phi}\left(x^{\prime}, t\right)\right),
\end{aligned}
$$

where we have used Eqs. (4) and (7). We note that Eq. (9) is closed in terms of $\hat{\phi}$, and $\Phi$ is determined by $\hat{\phi}$. To investigate the spatiotemporal patterns of $\phi$, we only have to solve Eq. (9) for $\hat{\phi}$.

Equations (8) and (9) always have the in-phase solution as a stationary solution where all oscillators are completely synchronized and their phase difference is 0 , i.e., $\Phi(t)=A(0) t$ and $\hat{\phi}(x, t)=0$. A linear stability analysis of the in-phase solution shows that the in-phase state is destabilized below the characteristic value of $k$ [3], given by

$$
k^{*}=A^{\prime}(0) / \pi^{2}
$$

where the corresponding eigenmode is $\cos \pi x$ (see Appendix A). In the parameter region $k<k^{*}$ various oscillation patterns can emerge, which we investigate in detail in the subsequent sections.

\section{NUMERICAL SIMULATIONS}

To investigate the behavior of $\hat{\phi}$ that obeys Eq. (9), we have numerically solved Eq. (9) for $\hat{\phi}$ (or, equivalently, we have solved Eq. (3) for $\phi$ and subtracted its mean value $\Phi$ from $\phi)$. The methods that we have used are as follows. First, we discretize Eq. (9) with respect to $x$ as $x=i / N(i=1, \ldots, N)$ and regard Eq. (9) as $N$ simultaneous differential equations for $\hat{\phi}(i / N, t)$, where the second derivative term in Eq. (9) has been discretized by the usual central difference method. Next, we solve the simultaneous differential equations by using the Runge-Kutta method to fourth order with an appropriate time increment.

The initial conditions taken here are $\hat{\phi}(x, 0)=0.1 x+$ $\xi(x)-\int_{0}^{1}\left(0.1 x^{\prime}+\xi\left(x^{\prime}\right)\right) d x^{\prime}$, where $\xi$ is a uniformly distributed random number in the range -0.01 to 0.01 . The term 


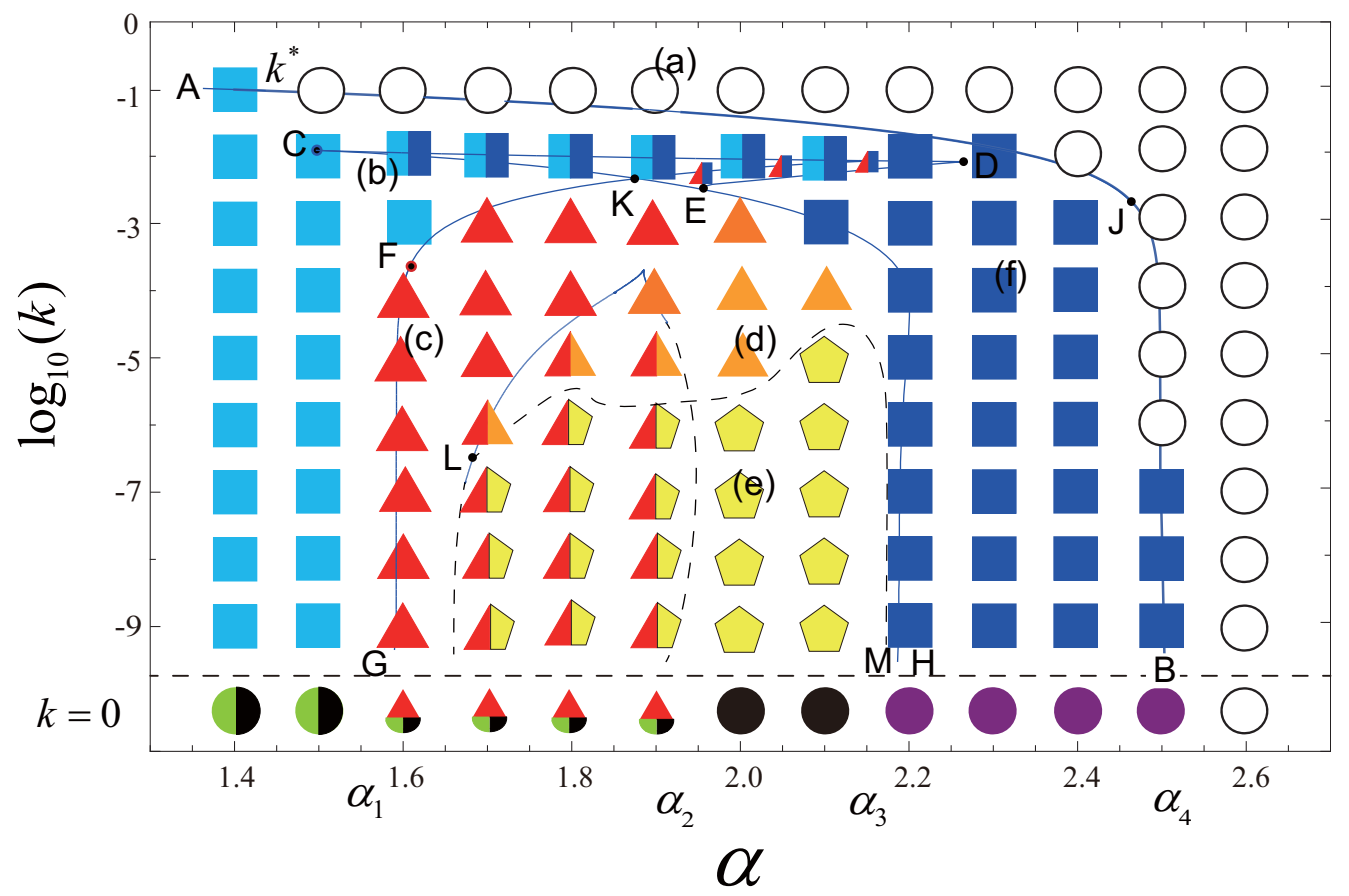

FIG. 1. (Color online) Phase diagram of the final state of $\hat{\phi}$ obeying Eq. (9) for $N=20$ and $r=0.4$. (i) White circles: spatially uniform state (in-phase state in $\phi$ ). (ii) Dark-blue and light-blue squares: monotonically increasing (or decreasing) states (traveling waves in $\phi$ ). In the dark-blue state the profile of $\hat{\phi}(x)$ is sigmoid-like, whereas in the light-blue state $\hat{\phi}$ exhibits a smoother and more spread-out shape [see Figs. 3(e) and 3(b)]. (iii) Red triangles: small-amplitude oscillations. (iv) Orange triangles: large-amplitude oscillations. (v) Yellow pentagons: locally unstable but globally attractive oscillations. (vi) Purple circles: stable two-cluster states. (vii) Black circles: locally unstable but globally attractive two-cluster states. (viii) Green circles: incoherent states. Curve AB indicates the values of $k=k^{*}$ below which the in-phase state is destabilized [see Eq. (10)]. Multicolored circles and polygons indicate the coexistence of two or more states. Explanations of each state are given in the text. Blue lines are the bifurcation curves of the system for $N=15$, which were computed using the software MATCONT [23], carrying out numerical continuation of the bifurcation points. MATCONT does not converge for some ranges of parameters, and dashed lines are guided by the colored polygons, i.e., the results of the numerical simulation for $N=20$. (Since the phase diagrams for $N=15$ and $N=20$ are almost the same, these lines are smoothly connected).

$0.1 x$ is used to apply a small gradient to this initial condition, which is done for the following reasons. The system described by Eq. (9) is completely symmetric with respect to $x=1 / 2$. Therefore, if the system would undergo symmetry breaking for some parameter values and we would start with nonbiased initial conditions, then increasing profiles of $\hat{\phi}$ and decreasing profiles of $\hat{\phi}$ would arise with equal probability. Since these increasing and decreasing profiles of $\hat{\phi}$ are equivalent as the state of the system, we avoid unnecessary complexity in our analysis, by applying a bias to the initial conditions such that the increasing profile of $\hat{\phi}$ easily appears. The integral term involved in the initial conditions ensures that $\hat{\phi}(x, 0)$ satisfies Eq. (7). The effects of the finite nature of $N$, which corresponds to the total number of oscillators, were examined by changing $N$ from 10 to 2000 . We found that in order to see the essential features of the solutions of Eq. (9), it is sufficient to consider the finite case of $N \geqslant 20$. Therefore, unless specifically stated otherwise, the total number of oscillators in our simulations is $N=20$. If a high accuracy is required, we can increase $N$. When the system reaches a steady state after a long simulation time, we refer to this as the final state of the system. The final states can include the oscillatory motion of $\hat{\phi}$. Sometimes it is more useful to explain the state by using $\phi$ instead of $\hat{\phi}$. In this situation, we refer to $\phi$ together with phrases such as "in the original oscillation system" or "in $\phi . "$

\section{A. Phase diagram of the final states of Eq. (9)}

Figure 1 shows the phase diagram of the final state of $\hat{\phi}$ obtained by numerically solving Eq. (9) for $N=20$ and $r=$ 0.4 . The colored circles and polygons indicate the types of final states for different values of $\alpha$ and $k$. Each state and its characteristics are explained in detail in the subsequent subsections. The topological structure of the phase diagram is essentially the same for all values of $r(0<r<1 / 2)$, so we use the value $r=0.4$ throughout this paper.

\section{B. Brief review of the final states of globally coupled oscillators}

Before giving a detailed explanation of each final state of Eq. (9), it is helpful to review some results for globally coupled oscillators, because the case of $k \rightarrow+0$ in Eq. (9) is in some way related to the global coupling case $(k=0)$. There are four characteristic values of $\alpha$ that separate the parameter domain into cases from which different final states arise [12]. These values are denoted by $\alpha_{1}, \alpha_{2}, \alpha_{3}$, and $\alpha_{4}$ (see Fig. 1 ). $\alpha_{4}$ is the value below which the in-phase state is unstable. The value of $\alpha_{4}$ is obtained by linear stability analysis of the in-phase state and is $\alpha_{4}=\arccos (-2 r)$ (Appendix A). In the region $\alpha_{3}<$ $\alpha<\alpha_{4}$, two-cluster states emerge, which are expressed as $\hat{\phi}_{1}=\hat{\phi}_{2}=\cdots=\hat{\phi}_{M}=\Phi_{1}$ and $\hat{\phi}_{M+1}=\hat{\phi}_{M+2}=\cdots=\hat{\phi}_{N}=$ $\Phi_{2}$, with some positive integer $M$ and constants $\Phi_{1}$ and $\Phi_{2}$. 

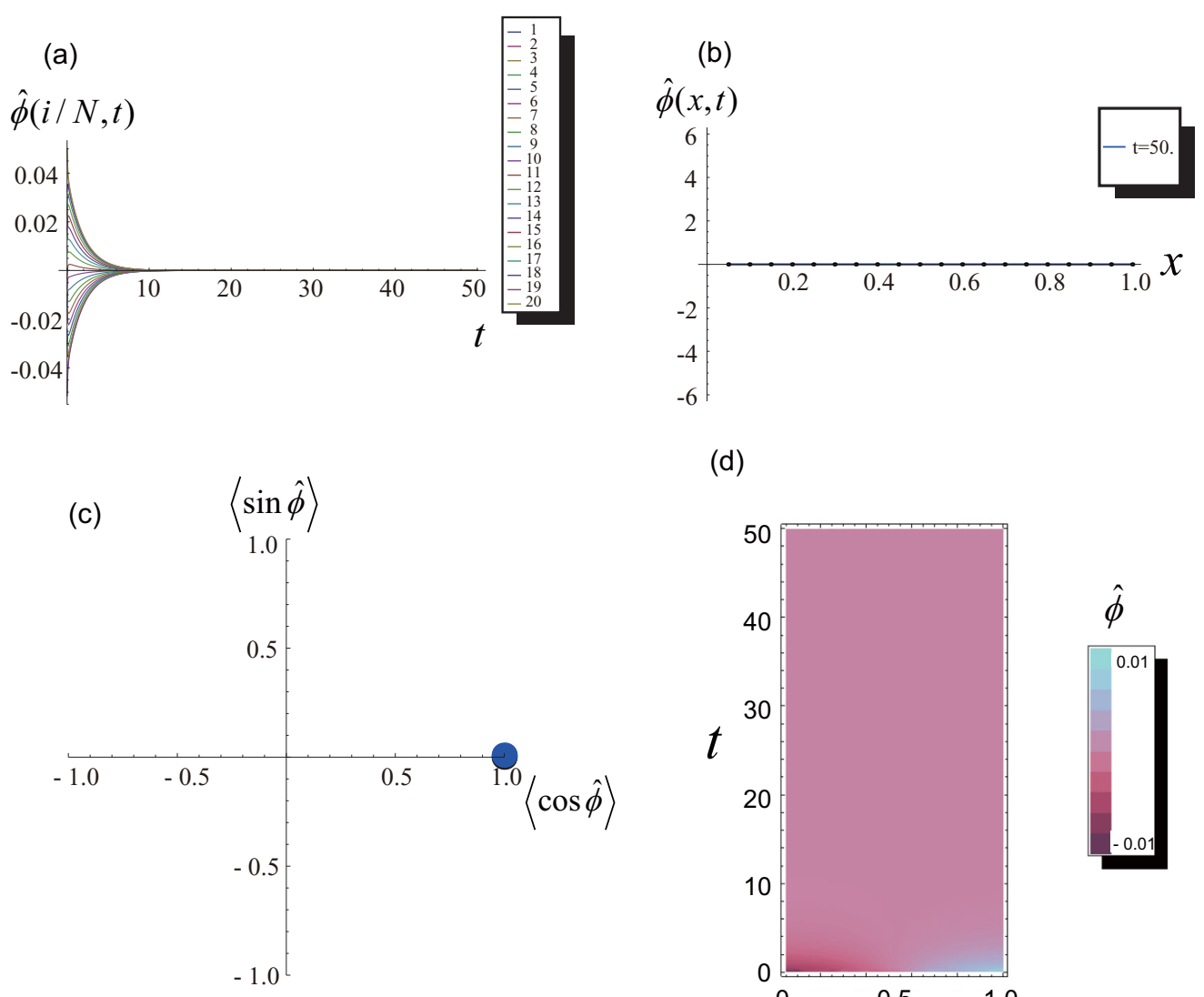

(d)

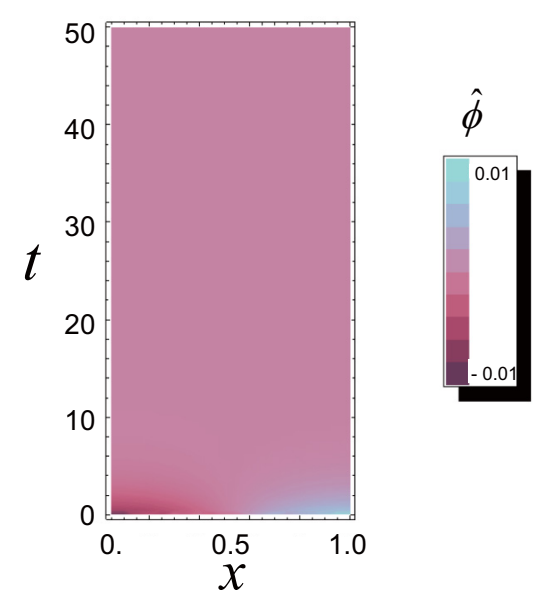

FIG. 2. (Color online) Aspects of the spatially uniform state of $\hat{\phi}$ (in-phase state in $\phi$ ). (a) Time series of $\hat{\phi}$. (b) Stationary profile of $\hat{\phi}$. (c) Trajectory of the state of the system in $\langle\cos \hat{\phi}\rangle-\langle\sin \hat{\phi}\rangle$ space, for which the black and blue circles represent the starting point and the state at some later time, respectively. (d) Kymograph (time-space plot) of $\hat{\phi}$. In Figs. 3, 5, 6, and 9, the aspects of each state are given in the same way. Parameters used here are $N=20, k=0.1, \alpha=1.9$, and $r=0.4$.

The main properties of these two-cluster states have been investigated previously [11] and are summarized in Appendix D. The value of $\alpha_{3}$ is determined by the linear stability analysis of the two-cluster state. The quantities $p=M / N$ and $\Delta=\Phi_{1}-\Phi_{2}$ are useful parameters to specify a two-cluster state. In the region of $\alpha_{2}<\alpha<\alpha_{3}$, locally unstable but globally attractive two-cluster states emerge [11], where the phrase "locally unstable but globally attractive" implies that if Eq. (9) with $k=0$ is linearized about the two-cluster state, it has eigenvalues with positive real parts, i.e., the two-cluster state is a "saddle." However, there are many such two-cluster states for a given $\alpha$ that are connected by attractive heteroclinic orbits. After a long simulation time, one saddle two-cluster state emerges as a stationary state due to rounding errors in the simulation [11]. If noise is continuously added to this system, it exhibits noise-driven periodic motion, referred to as slow switching $[11,13,14]$. In the region $\alpha_{1}<\alpha<\alpha_{2}$, oscillation of the relative phase difference arises with no noise. The values of $\alpha_{1}$ and $\alpha_{2}$ can be numerically determined. In the region $\alpha<\alpha_{1}$ an incoherent state (green) emerges, which is the state where the values of $\hat{\phi}$ are homogeneously distributed and is expressed as $P(\hat{\phi}, t)=1 / 2 \pi$ with a distribution function of $\hat{\phi}, P[11]$. In the parameter regions $\alpha_{1}<\alpha<\alpha_{2}$ and $\alpha<\alpha_{1}$, other states coexist. This situation is represented by multicolored circles in Fig. 1. Although the state that is realized depends on the initial conditions, the vast majority of initial conditions result in the state described above.

\section{Case of a spatially uniform state of $\hat{\phi}$ (in-phase state in $\phi$ ) (white circles)}

Figure 2 shows the time series and profile of $\hat{\phi}$ in the case where the final state is spatially uniform, i.e., $\hat{\phi}(x)=0$ (in-phase state in $\phi$ ). We also plot some other quantities that characterize the state of the system, given by $\langle\cos \hat{\phi}\rangle=\int_{0}^{1} \cos (\hat{\phi}(x, t)) d x,\langle\sin \hat{\phi}\rangle=\int_{0}^{1} \sin (\hat{\phi}(x, t)) d x$. We refer to these quantities and their plots as the aspects of the state under consideration. As stated above, when $k<k^{*}$ the in-phase state becomes unstable. We see from the symmetry of the system that destabilization of the in-phase state occurs in the form of a pitchfork bifurcation. In general, this pitchfork bifurcation can be of either the supercritical or the subcritical type. The type that is realized can be analytically investigated by reductive perturbation [15] to the in-phase solution in the neighborhood of the critical point $k=k^{*}$ (Appendix $\left.\mathrm{B}\right)$. The analytical results show that when $\frac{A^{\prime \prime}(0)^{2}}{24 A^{\prime}(0)}+\frac{3}{8} A^{\prime \prime \prime}(0)<0$ the bifurcation is supercritical, whereas when $\frac{A^{\prime \prime}(0)^{2}}{24 A^{\prime}(0)}+\frac{3}{8} A^{\prime \prime \prime}(0)>0$ it is subcritical. For the case of $r=0.4$, the characteristic value of $\alpha$ that separates the 

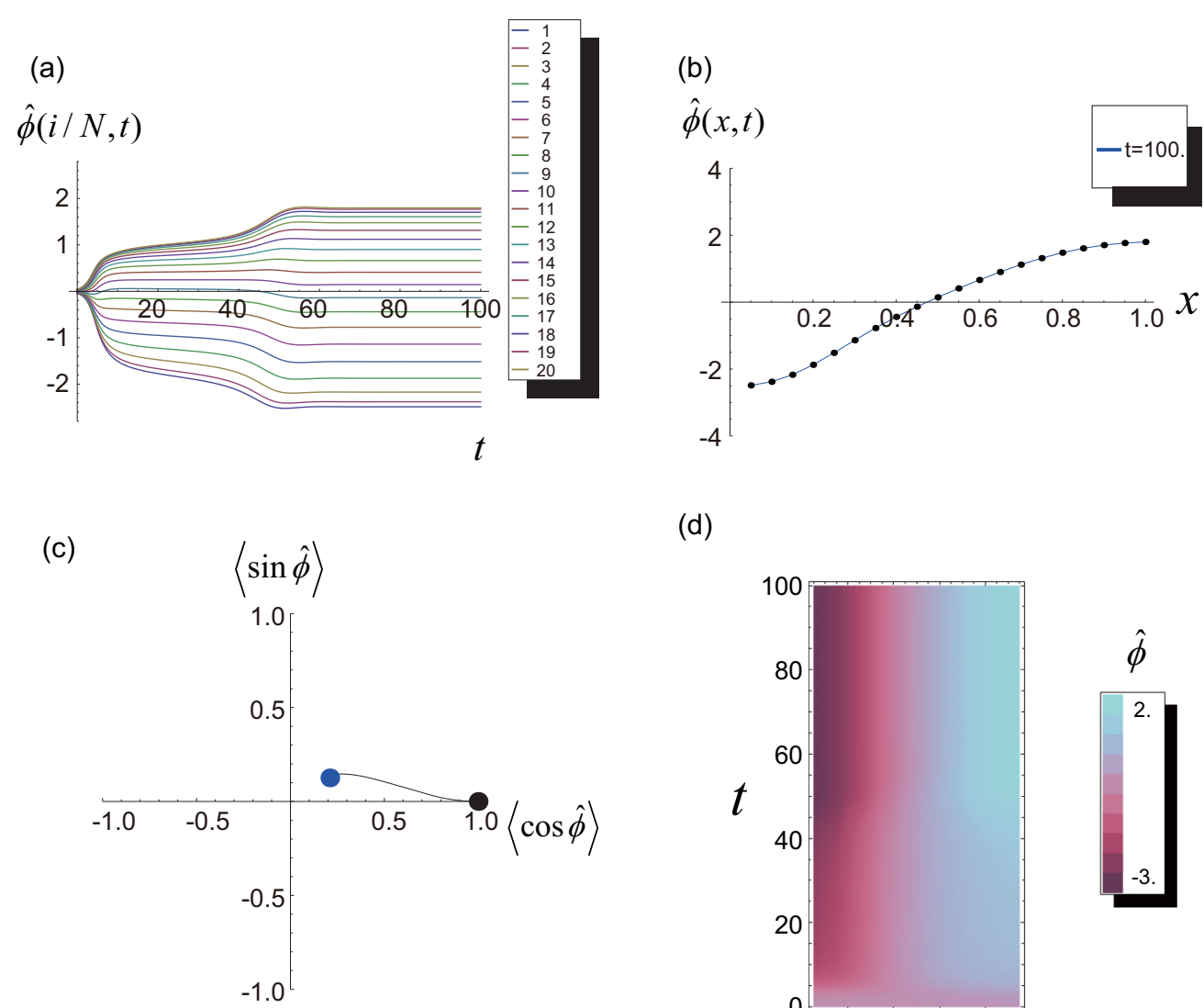

(d)

(e)
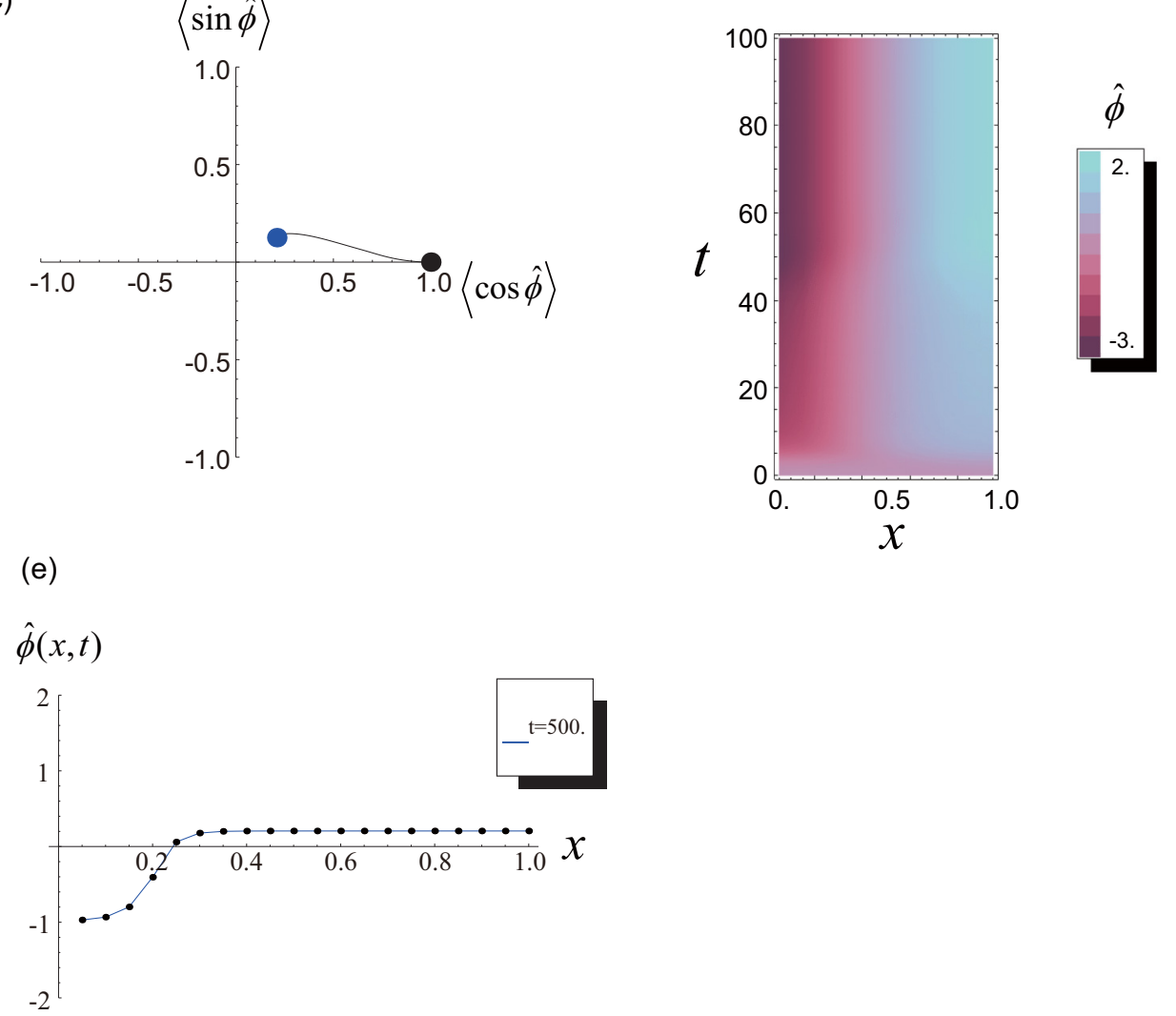

FIG. 3. (Color online) (a-d) Aspects of the monotonically increasing state of $\hat{\phi}$ (traveling wave in $\phi$ ) for $N=20, k=0.01, \alpha=1.6$, and $r=0.4$, parameters that correspond to point (b) in Fig. 1. (e) Stationary profile of $\hat{\phi}$ for $N=20, \alpha=2.3, k=10^{-4}$, and $r=0.4$, parameters that correspond to point (f) in Fig. 1.

supercritical and subcritical regions is 2.47 , which is indicated by point (j) in Fig. 1. This is in agreement with numerical simulations.

\section{Case of monotonically increasing (or decreasing) states of $\hat{\phi}$ (traveling waves in $\phi$ ) (dark-blue and light-blue squares)}

Below the critical value of $k$, which is $k^{*}$, a monotonically increasing (or decreasing) stationary profile of $\hat{\phi}(x)$ emerges. This state corresponds to a traveling wave in the original oscillator system because, if we represent this state by $\phi$, we have $\phi(x, t)=\omega_{0} t+\hat{\phi}(x)$, with a constant $\omega_{0}$ obtained from Eq. (8) as $\omega_{0}=\int_{0}^{1} d x \int_{0}^{1} d x^{\prime} A\left(\hat{\phi}(x)-\hat{\phi}\left(x^{\prime}\right)\right)$. Figure 3 shows the aspects of $\hat{\phi}$ in the monotonically increasing states, of which there are two types. One type is the state that appears in the parameter region $\alpha<\alpha_{1}$, indicated by light-blue squares in Fig. 1. A typical profile of this state is given in Fig. 3(b). A characteristic of this profile is that the slope of $\hat{\phi}(x)$ is relatively unmodulated; i.e., the second-order derivative of $\hat{\phi},\left|d^{2} \hat{\phi} / d x^{2}\right|$, is small, and the distribution of $\hat{\phi}$ is homogeneously spread. These characteristics arise from the fact that for $\alpha<\alpha_{1}$ and $k=0$ the incoherent state is realized. The other monotonically increasing state appears in the parameter region indicated by dark-blue squares in Fig. 1. A typical profile of this state is given in Fig. 3(e) and has a sigmoid-like shape; i.e., two plateau regions appear in $\hat{\phi}(x)$ that are connected by a 


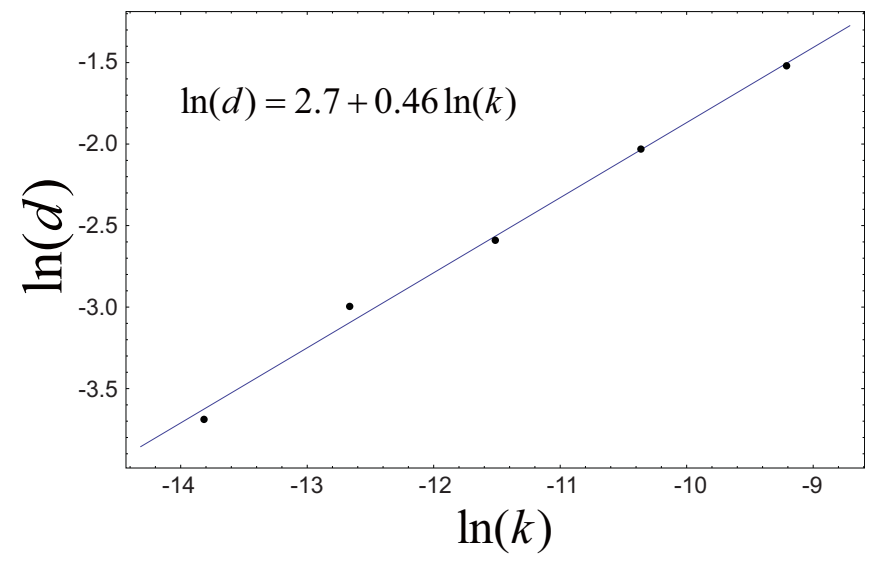

FIG. 4. (Color online) The $k$ dependence of the width $d$ of the interface between two plateau domains in $\hat{\phi}$ for $N=320, \alpha=2.3$, and $r=0.4$ (blue region in Fig. 1). The width $d$ is determined in the following way. First, we define $\Phi_{1}$ and $\Phi_{2}$ as $\hat{\phi}(1)=\Phi_{1}$ and $\hat{\phi}(0)=\Phi_{2}$. Next, we determine the positions $x=x_{1}$ and $x_{2}$ such that $\hat{\phi}\left(x_{1}\right)=\left(\Phi_{1}+\Phi_{2}\right) / 2+\delta$ and $\hat{\phi}\left(x_{2}\right)=\left(\Phi_{1}+\Phi_{2}\right) / 2-\delta$, where $\delta=0.95\left(\Phi_{1}-\Phi_{2}\right) / 2$. Then $d$ is given by $d=x_{1}-x_{2}$. We see that $d$ is nearly proportional to $k^{1 / 2}$.

smooth interface due to the local coupling. This shape of $\hat{\phi}$ reflects that for $k=0$ and $\alpha_{3}<\alpha<\alpha_{4}$, stable two-cluster states emerge. We refer to the former monotonically increasing state as the light-blue or smoothly increasing state and to the latter state as the dark-blue or shelf-shaped state.

These dark-blue and light-blue states coexist in the parameter region indicated by curve CKD in Fig. 1. Point $\mathrm{C}$ is the cusp point. In region CKD, the state that is realized depends on the initial condition.

We have measured the width $d$ of the interface appearing in the dark blue state as a function of $k$ and found that $d$ is approximately proportional to $\sqrt{k}$ (see Fig. 4), which implies that the characteristic length of the system is determined by the coefficient $k$ in Eq. (9) when $A$ is given.

As described in Appendix D, when $k=0$ there are an infinite number of possible two-cluster states in the parameter region $\alpha_{3}<\alpha<\alpha_{4}$. That is, the parameters that specify the state, $p$ and $\Delta$, are not uniquely determined. However, for $k>0$ the profile of $\hat{\phi}(x)$ is uniquely determined. This fact indicates that the degeneracy of the globally coupled state is resolved by the local coupling term in Eq. (3). The unique selection of the values of $p$ and $\Delta$ is discussed from an analytical point of view in Appendix E.

\section{E. Case of two stable oscillations in $\hat{\phi}$ (red and orange triangles)}

When $k$ becomes smaller than some characteristic value, the monotonically increasing states are destabilized and stable oscillations of $\hat{\phi}$ emerge. The parameter region in which these oscillations occur is indicated by red and orange triangles in Fig. 1. There are four ways in which oscillations emerge in this system: (i) On curve FD in Fig. 1 a supercritical Hopf bifurcation occurs, in which the light-blue state is destabilized. (ii) On curve EH an infinite period bifurcation [16] occurs, which is sometimes known as a saddle-node homoclinic bifurcation, where the dark-blue state is destabilized. (iii) On curve DE the system undergoes homoclinic bifurcation [16], in which the oscillation of $\hat{\phi}$ indicated by the red triangles collides with a saddle state and disappears. (iv) On curve FG the system undergoes subcritical Hopf bifurcation, in which the light-blue state is destabilized.

There are two types of oscillations in this parameter region. One occurs at the parameters indicated by red triangles, whose characteristic is that the amplitude of $\hat{\phi}(x, t)$ at any $x$ is small; hence we refer to this as the small-amplitude oscillation. The other type of oscillation occurs at the parameters indicated by orange triangles, in which the change in $\hat{\phi}(x, t)$ with respect to $t$ is relatively large; hence we refer to this as the large-amplitude oscillation. The two types of oscillations have different origins and properties, which are explained in detail below.

\section{Small-amplitude oscillation in $\hat{\phi}$ (red triangles)}

Figure 5 shows the aspects of the small-amplitude oscillation. One characteristic of this oscillation is that the wave form of the oscillation is longitudinal, which is seen in the kymograph of $\hat{\phi}$ in Fig. 5(d). This characteristic reflects the fact that the light-blue state has already undergone symmetry breaking. In other words, if we take another form of the light-blue state, i.e., a monotonically decreasing state, the direction of the propagation of the wave becomes opposite.

The origin of this oscillation lies in the global coupling $A$, which is confirmed by the operation $k \rightarrow+0$ in the numerical simulations of Eq. (9), in which the small-amplitude oscillation for $k=+0$ coincides with the $k=0$ oscillation. The local coupling term in this oscillation orders the phases.

It is interesting to see this small-amplitude oscillation in the original oscillation system using $\phi$, in which two types of wave propagation are observed [see Fig. 5(e)]. To see this from an analytical point of view, we focus on the vicinity of curve $\mathrm{KF}$, in which a supercritical Hopf bifurcation occurs. In this area $\phi$ is expressed as

$$
\phi(x, t) \simeq \omega_{1} t+\phi^{(0)}(x)+a(x) \cos \left(\omega_{0} t-b(x)\right),
$$

where $\phi^{(0)}(x)$ is the profile of the light-blue state for parameters on curve $\operatorname{KF}\left(d \phi^{(0)} / d x>0\right)$. Here $\omega_{0}$ is a positive constant, $a$ and $b$ are functions of $x$ that satisfy $a(x)>0,\left.a^{\prime}(x)\right|_{x=0,1}=0$, and $\left.b^{\prime}(x)\right|_{x=0,1}=0 . b^{\prime}(x)$ tends to be positive for $0<x<$ 1. $\omega_{1}=\int_{0}^{1} d x \int_{0}^{1} d x^{\prime} A\left(\hat{\phi}^{(0)}(x)-\hat{\phi}^{(0)}\left(x^{\prime}\right)\right)$. Under periodic boundary conditions the forms of $a$ and $b$ are explicitly given (see Appendix C). Since $b^{\prime}(x)>0$ for $0<x<1$, a traveling wave runs to the right, which comes from the cosine term in Eq. (11). Furthermore, since $\phi^{(0)^{\prime}}(x)>0$, another traveling wave runs to the left, which comes from the $\omega_{1} t+\phi^{(0)}(x)$ term in Eq. (11). We note that if we choose $\hat{\phi}^{(0)}(1-x)$ as the stationary state for the parameters on curve KF, both directions of the two traveling waves become opposite.

\section{Large-amplitude oscillation in $\hat{\phi}$ (orange triangles)}

In the parameter region indicated by orange triangles in Fig. 1 another type of oscillation emerges, termed the large-amplitude oscillation. The aspects of this oscillation are given in Fig. 6 and the characteristics of this state are as follows. (i) This oscillation originates from the combination of local and global coupling, which is understood from the 
(a)
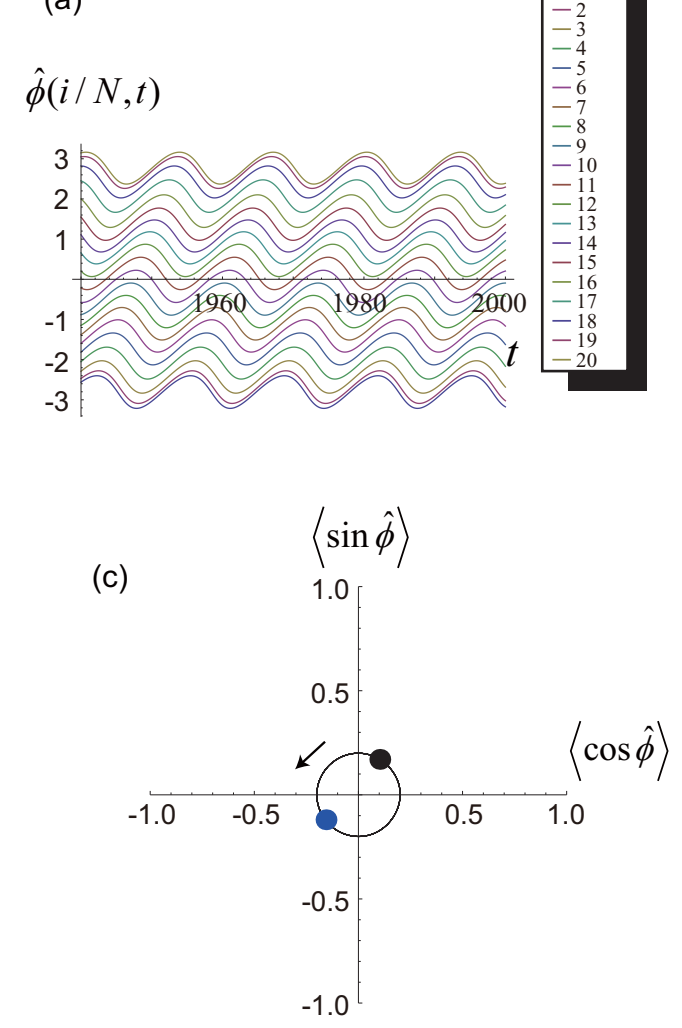

(e)

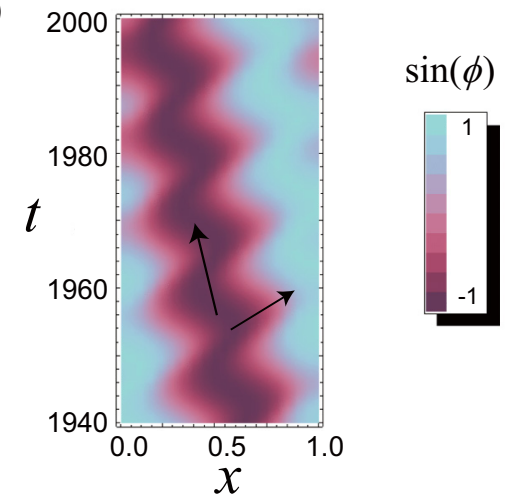

(b)

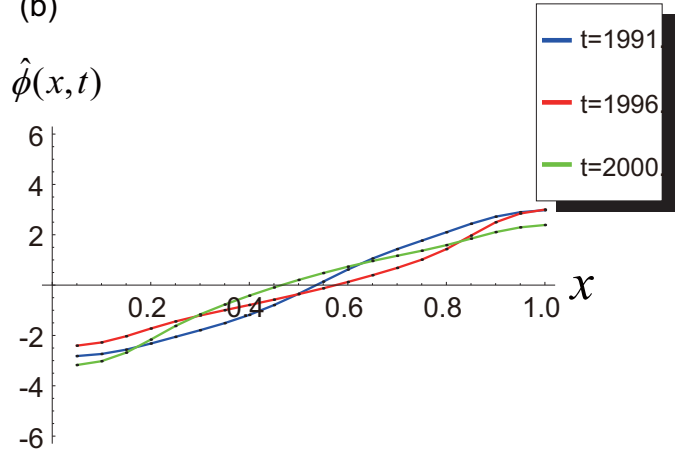

(d)

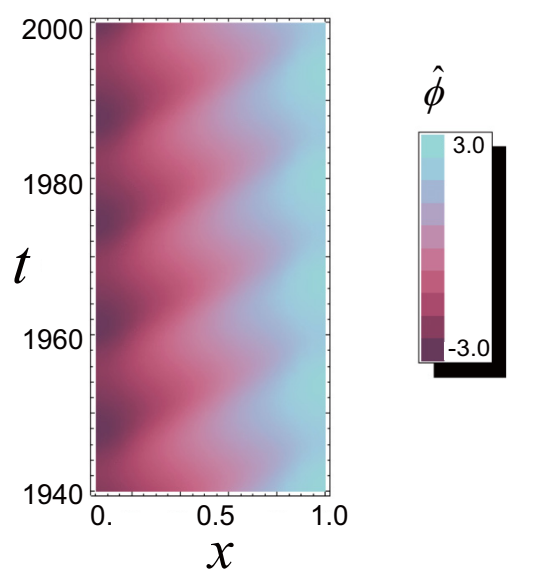

FIG. 5. (Color online) (a-d) Aspects of the small-amplitude oscillations of $\hat{\phi}$ occurring at $k=10^{-5}, \alpha=1.6$, and $r=0.4$ with $N=20$. This type of oscillation shows a longitudinal wave as apparent in (a), (b), and (d). If we look at this oscillation in terms of the original system, i.e., in terms of $\phi(x, t)$, we find the propagation of two traveling waves [arrows in (e)].

fact that both for $k=0$ and for $k \gg 1$ this type of oscillation does not occur. (ii) During the periodic motion of the system the profile of $\hat{\phi}$ maintains a shelf-like shape (sigmoid-like shape); i.e., two plateau regions appear in $\hat{\phi}$ [see Fig. 6(b)]. Changing the ratio of the size of each domain in $\hat{\phi}$ causes the system to oscillate. (iii) As $k$ approaches curve $\mathrm{EH}$ from below in Fig. 1, denoted $k_{1}$, the period $T$ of the oscillation diverges as $T \propto\left(k_{1}-k\right)^{-1 / 2}$ [see Fig. 7 and inset (b) there]. This implies that at $k=k_{1}$ an infinite period bifurcation (saddle-node homoclinic bifurcation) occurs. (iv) As $k \rightarrow+0$, the period $T$ of the oscillation diverges since $T \propto k^{-1 / 2}$ [see inset (a) in Fig. 7]. These characteristics, (i)-(iv), are related to the origin of occurrence of the oscillation, so we now move on to an explanation of this issue.

\section{Mechanism of occurrence of large-amplitude oscillation}

As mentioned in Sec. III B, for $k=0$ and $\alpha_{2}<\alpha<\alpha_{3}$, locally unstable but globally attractive two-cluster states emerge (black circles in Fig. 1). These states are expressed as

$$
\hat{\phi}(x)=\Phi_{2} \Theta((1-p)-x)+\Phi_{1} \Theta(x-(1-p)),
$$

where $\Phi_{1}$ and $\Phi_{2}$ are the phases of each domain, and $p$ is the fraction of the cluster that has phase $\Phi_{1}$ with respect to that of the whole system. $\Theta(x)$ is a Heaviside function defined as $\Theta(x)=0$ for $x<0$ and $\Theta(x)=1$ for $x>0$. The phase difference between two clusters is defined as $\Delta=\Phi_{1}-\Phi_{2}$. As mentioned in Sec. III B, there are many two-cluster states 


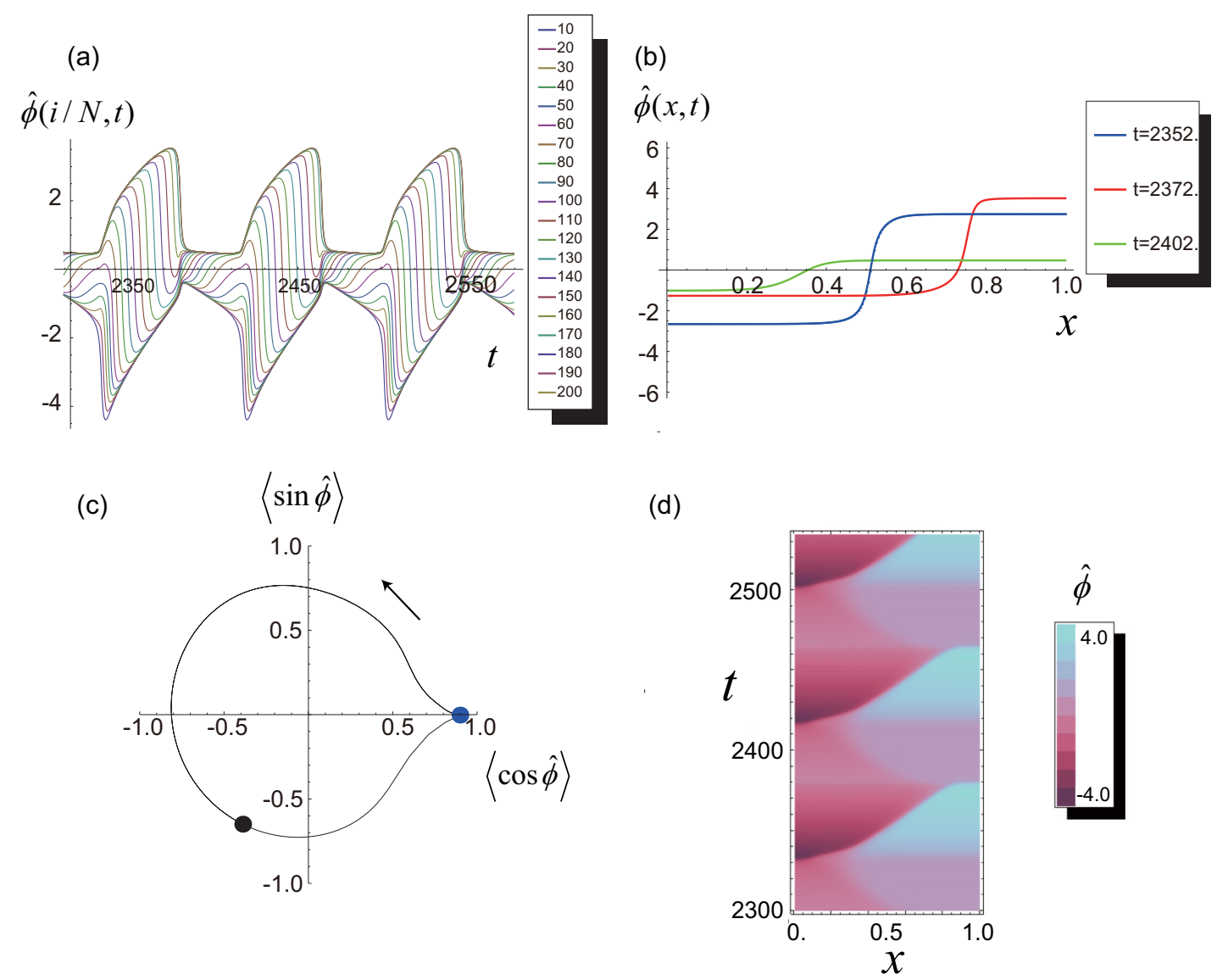

FIG. 6. (Color online) Aspects of the large-amplitude oscillation occurring for $k=10^{-5}, \alpha=2.0$, and $r=0.4$ with $N=200$. The appearance of this oscillation is due to the combination of global coupling, which tends to form two-cluster states, and local coupling, which connects clusters smoothly (see Sec. IIIE 3).

for a given $\alpha$, and these states are in fact locally unstable. That is, if we perform a linear stability analysis of this state, we obtain eigenvalues with positive real parts; these states are destroyed by any small perturbations. However,

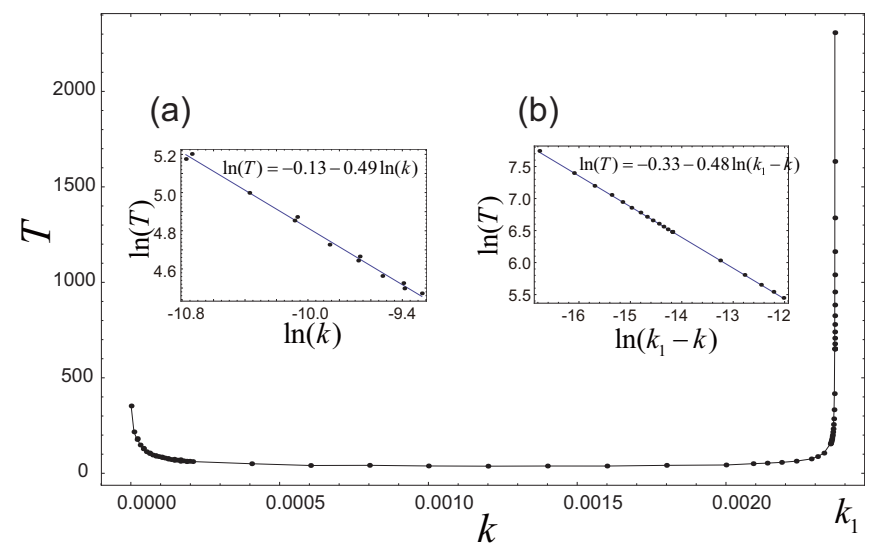

FIG. 7. (Color online) Period $T$ of the large-amplitude oscillation as a function of $k$ for $N=160, \alpha=2.0$, and $r=0.4$. Inset (a): A $\log -\log$ plot of the period for $k \ll 1$. Inset (b): A log-log plot of the period with respect to $\left(k_{1}-k\right)$, where $k_{1}$ is a characteristic value of $k$ at which the monotonically increasing state is destabilized. Insets (a) and (b) show that period $T$ behaves as $T \propto\left(k_{1}-k\right)^{(-1 / 2)}$ near $k=k_{1}(=0.00236)$ and $T \propto k^{(-1 / 2)}$ near $k=0$, respectively. these two-cluster states are connected to other two-cluster states by attractive heteroclinic orbits [11], and hence after a long simulation time one of the saddle two-cluster states is realized as an apparently stationary state. Here we use "apparently" to mean that the realized two-cluster state is not exactly stationary; its stationary nature is the result of rounding errors in the numerical simulations. Nevertheless, no matter how accurately the numerical simulations are performed, the system reaches a two-cluster state within a finite time as long as the accuracy of the numerical simuation is finite. In that sense the two-cluster state is apparently stationary [11]. The necessary conditions for the two-cluster states to be apparently stationary are threefold: Equation (D3) must be satisfied, and $\lambda_{1} \lambda_{2}<0$ and $\lambda_{3}<0$ must hold, where $\lambda_{i}$ ( $i=1,2,3)$ are eigenvalues of the linearized equation about the two-cluster state; its meanings and explicit forms are given in Eq. (D4). Equation (D3) gives the relationship between $p$ and $\Delta$, which is represented by an $N$-shaped curve in the $p$ - $\Delta$ plane (see Fig. 8). This $N$-shaped curve consists of three types of branches, which are classified by the following conditions:

$$
\begin{aligned}
& \lambda_{1}<0, \quad \lambda_{2}>0, \quad \lambda_{3}<0 \quad \text { (blue branch), } \\
& \lambda_{3}>0 \text { (gray branch), } \\
& \lambda_{1}>0, \quad \lambda_{2}<0, \quad \lambda_{3}<0 \quad \text { (red branch). }
\end{aligned}
$$




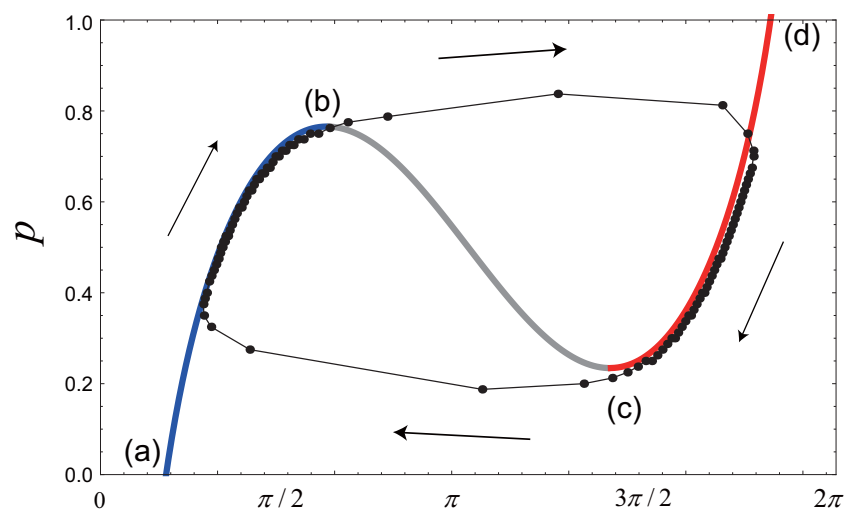

$\Delta$

FIG. 8. (Color online) Trajectory of the system in $p-\Delta$ space for $N=80, k=10^{-5}, \alpha=2.0$, and $r=0.4$ (black circles and curve), which describes the mechanism of the large-amplitude oscillation (see also Sec. III E 3). The values of $p$ and $\Delta$ of $\hat{\phi}$ for $k>0$ are evaluated such that $\hat{\phi}(1-p)=0$ and $\Delta=(1 / p) \int_{1-p}^{1} \hat{\phi}(x) d x-(1 /(1-$ p)) $\int_{0}^{1-p} \hat{\phi}(x) d x$. The $N$-shaped curve represents the relationship between $p$ and $\Delta$ [Eq. (D3)] that two-cluster states must satisfy in order to be stationary for $k=0$. This curve consists of three branches $[(a, b)$ blue, $(b, c)$ gray, and (c, d) red], the meaning and classification of which are explained in Eq. (13).

Since both inequalities, $\lambda_{1} \lambda_{2}<0$ and $\lambda_{3}<0$, must hold in order for the two-cluster state to be apparently stationary, only the states on the blue and red branches are candidates for this apparently stationary state.

If the local coupling in Eq. (9) is switched on; i.e., if $k>0$, a smooth interface that connects the two clusters is created and the two-cluster state is no longer even apparently stable because the interface applies a finite perturbation to the unstable two-cluster state. That is, the profiles of $\hat{\phi}$ begin to move. The direction of movement of the profile is such that a stable domain (cluster) in $\hat{\phi}$ expands and an unstable domain shrinks. According to the linear stability analysis of the two-cluster state [Eq. (13)], when the state is on the blue branch, the cluster with phase $\Phi_{1}$ is stable and the cluster with phase $\Phi_{2}$ is unstable. Thus, if we start with the state on the blue branch with $0<k \ll 1$, the system moves as outlined below.

Since in the blue branch the cluster with $\Phi_{1}$ is stable and the cluster with $\Phi_{2}$ is unstable, the system moves along the blue branch in such a way that $p$ increases (see Fig. 8). At some time the system reaches point (b), where there is no other branch that contains possible two-cluster states, so the system has to jump to the red branch. After arrival at the red branch the system moves oppositely along the red branch in such a way that $p$ decreases, because in the red branch the cluster with $\Phi_{1}$ is unstable and that with $\Phi_{2}$ is stable [see Eq. (13)]. After arrival at point (c) the system again jumps to the blue branch and returns to its original starting point, i.e., the trajectory is closed. In this way a cyclic motion occurs. Indeed, a numerical simulation performed for $N=80, \alpha=2.0 k=$ $10^{-5}$, and $r=0.4$, parameters which are in the orange-triangle region in Fig. 1, traces the trajectory indicated by the black curve and circles in Fig. 8. This is the mechanism by which the large-amplitude oscillation occurs.

If we understand this oscillation mechanism, the characteristics of this oscillation as described in Sec. IIIE2 are easily understood. For example, characteristic (iv) is interpreted as follows. As mentioned above, the driving force that moves the profile of $\hat{\phi}$ for $k>0$ is the interface between two-cluster domains. It is thus natural to envisage that the rate of change of the profile of $\hat{\phi}$ is proportional to some characteristic quantity of the interface, for example, its width. Since the width of the interface is proportional to $k^{1 / 2}$ (see Fig. 4), it is reasonable to expect that the period of the oscillation, $T$, is proportional to $k^{-1 / 2}$.

\section{F. Case of locally unstable but globally attractive oscillation of $\hat{\phi}$ (yellow pentagons)}

When $k$ decreases further in the region $\alpha_{2}<\alpha<\alpha_{3}$, the large-amplitude oscillation loses its stability and another type of oscillation emerges (yellow pentagons in Fig. 1). This oscillation has the property that it is locally unstable but globally attractive. The phrase "locally unstable" means that when Eq. (9) is linearized about this oscillation, it possesses Floquet exponents with positive real parts or, equivalently, positive Lyapunov exponents. That is, even extremely small perturbations destroy the periodic motion (see Fig. 9). However, the perturbed system necessarily returns to the original periodic motion after a finite time interval, which is demonstrated in Fig. 9. We describe this oscillation as locally unstable but globally attractive. Even under large perturbations the system always returns to the original periodic orbit after a finite time interval, thus the global stability of the oscillation is very strong. We can distinguish the locally unstable but globally attractive oscillation from the large-amplitude oscillation by calculating the largest Lyapunov exponent of the oscillation [17]. The dashed curve LM indicates the boundary between the orange-triangle and the yellow-pentagon regions for $N=20$ and $r=0.4$.

The local instability of this oscillation results from the smallness of $k$. Our scenario is as follows. As described in Sec. IIIE3, a two-cluster state is realized for $k=0$ and $\alpha_{2}<\alpha<\alpha_{3}$, in which one of the two clusters is unstable. For $k>0$ a smooth interface between the two clusters is created by the local coupling and it drives the movement of the profile of $\hat{\phi}$, which leads to the large-amplitude oscillation. In this situation the local coupling also plays another role, stabilization of the unstable cluster. That is, the attractive local coupling in Eq. (9) suppresses the instability of the unstable cluster in $\hat{\phi}$. However, if the value of $k$ becomes too small, the tendency towards stabilization of the local coupling is weakened and the cluster once again becomes unstable, while the driving force from the interface is still present. Local instability of this oscillation then arises. Indeed, only those unstable domains for $k=0$ show disturbance due to perturbations [see the profiles at $t=96$ and 378 in Fig. 9(b)].

On the other hand, it is difficult to explain the global stability of this oscillation; the numerical simulations suggest that the saddle oscillation connects with attractive homoclinic orbits. That is, unstable and stable invariant manifolds extending from the periodic orbit intersect each other in some region of the 

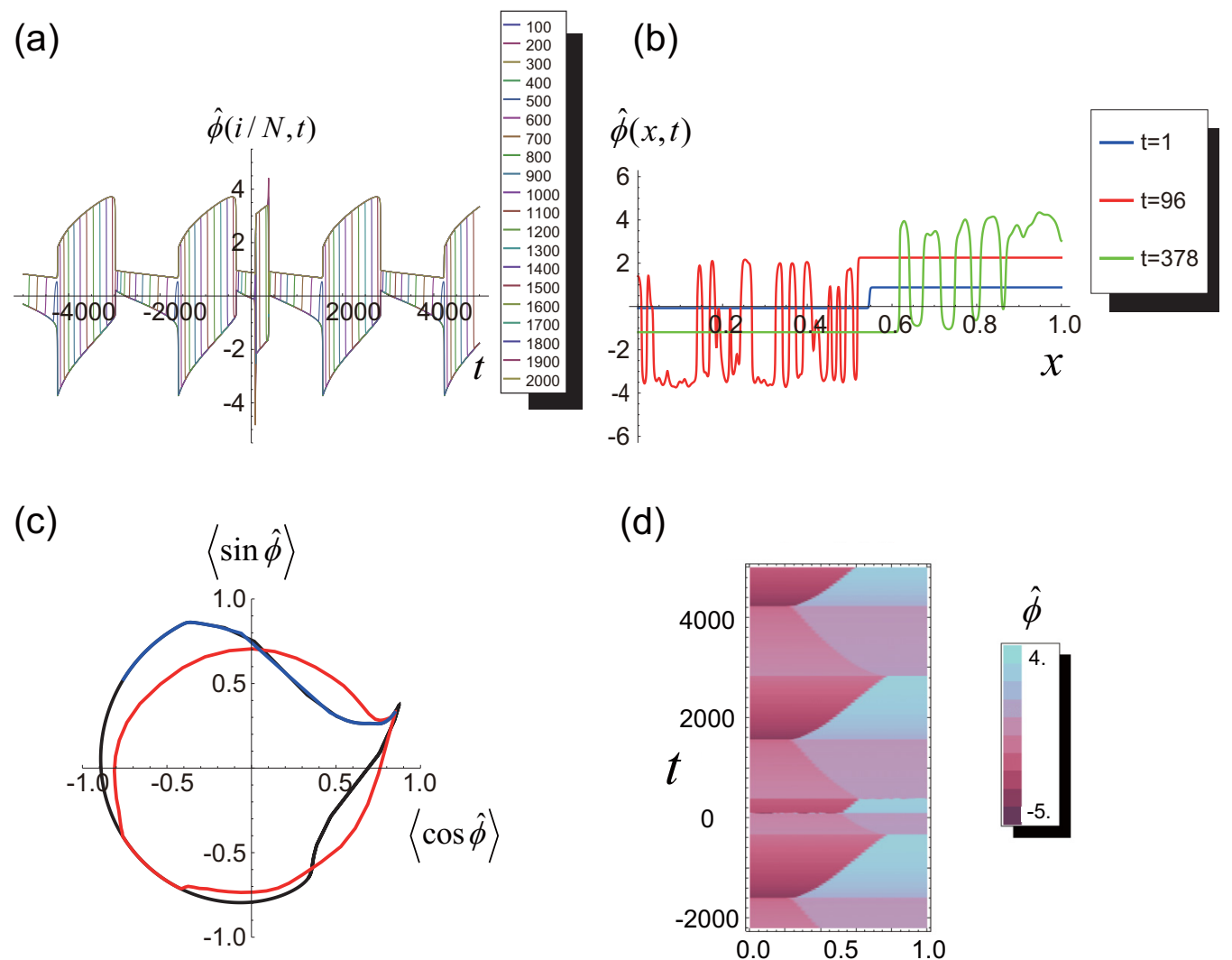

(d)

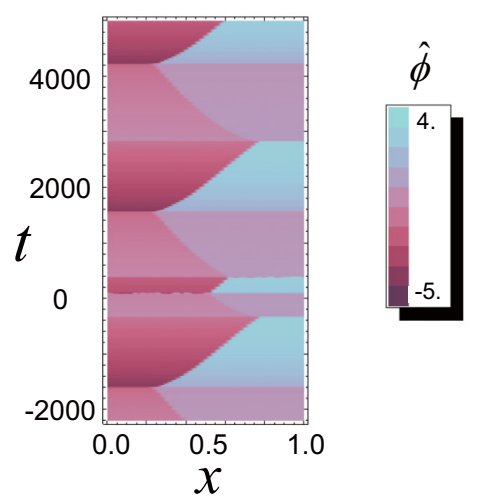

FIG. 9. (Color online) (a-d) Aspects of the locally unstable but globally attractive oscillation for $N=2000, k=10^{-7}, \alpha=2.0$, and $r=0.4$, parameters that correspond to point (e) in Fig. 1. Extremely low noise $\left(|\delta \hat{\phi}|<10^{-10}\right)$ was added to the periodic motion of the system after a long transient time (at $t=0$ ). We see that the stationary oscillation is destroyed by the small perturbation and recovers after some time interval. In (c) the trajectory of the system is indicated by three colors: black shows the trajectory for $t<0$; red, the trajectory for $0<t<400$; and blue, the trajectory for $400<t<1700$. This simulation was performed with quadruple precision implemented on the gcc compiler, i.e., using the _float128 floating type and corresponding math functions such as sinq().

phase space. This structure is closely related to the fact that for $k=0$ the saddle two-cluster states are connected with attractive heteroclinic orbits, achieving apparent stationary characteristics. The detailed structure surrounding the periodic orbit of this state is still unknown, and more detailed investigations are required.

\section{DISCUSSION AND CONCLUSIONS}

In the present work we have investigated locally attractively and globally repulsively coupled oscillators in one dimension using a phase model given by Eq. (9) [or, equivalently, Eq. (3)]. We have shown that, depending on the relative strengths of the local and global couplings and on the form of the global coupling $A$, various patterns of the phases emerge.

One of the characteristics of the system to be emphasized is that double traveling waves appear for the small-amplitude oscillation [Fig. 5(e)]. This behavior might be observed in experimental systems. Indeed, for the auto-oscillation of muscle, in which a normal type of traveling wave in the myofibril has already been reported [1], another oscillation mode is sometimes observed simultaneously [18], which might be related to the double traveling waves possessed by locally and globally coupled oscillators. The locally unstable but globally attractive oscillation of relative phase difference indicated by yellow pentagons in Fig. 1 represents another interesting mode of behavior of Eq. (9), which may also be of interest from a mathematical point of view. Some investigators have suggested the possibility that a saddle oscillation connected with attractive homoclinic orbits may exist [19,20]. However, there are few examples of this [20]. Equation (9) [or, equivalently, Eq. (3)] may be the first partial differential equation to show this type of oscillation. Using this equation, we can investigate how the spectrum of Lyapunov exponents of this oscillation is distributed and we might be able to clarify why and how this oscillation is globally stable. More detailed investigations of this topic are expected.

\section{ACKNOWLEDGMENTS}

We would like to thank Y. Kuramoto, Y. Kawamura, M. Toda, K. Ikeda, H. Wada, and Y. Shimizu for valuable comments and discussions. This work was supported by JSPS KAKENHI Grant N. 26400441 (to K.S).

\section{APPENDIX A: LINEAR STABILITY ANALYSIS OF THE STATIONARY SOLUTION OF EQ. (9)}

Let $\hat{\phi}(x, t)=\hat{\phi}^{(0)}(x)$ be the stationary solution of Eq. (9). By substituting $\hat{\phi}(x, t)=\hat{\phi}^{(0)}(x)+\psi(x, t)$ into Eq. (9), then 
linearizing Eq. (9) with respect to $\psi$, we obtain

$$
\begin{aligned}
\frac{\partial \psi(x, t)}{\partial t}= & k \frac{\partial^{2} \psi(x, t)}{\partial x^{2}}+\int_{0}^{1} d x^{\prime} A^{\prime}\left(\hat{\phi}^{(0)}(x)-\hat{\phi}^{(0)}\left(x^{\prime}\right)\right)\left(\psi(x, t)-\psi\left(x^{\prime}, t\right)\right) \\
& -\int_{0}^{1} d x \int_{0}^{1} d x^{\prime} A^{\prime}\left(\hat{\phi}^{(0)}(x)-\hat{\phi}^{(0)}\left(x^{\prime}\right)\right)\left(\psi(x, t)-\psi\left(x^{\prime}, t\right)\right) \\
= & : \int_{0}^{1} d x^{\prime} L\left(x, x^{\prime}\right) \psi\left(x^{\prime}, t\right),
\end{aligned}
$$

where we have used $\int_{0}^{1} \hat{\phi}^{(0)}\left(x^{\prime}\right) d x^{\prime}=0 . \int_{0}^{1} d x^{\prime} L\left(x, x^{\prime}\right)$ is the linear operator acting on any function of $x^{\prime}$ and $t$, defined above. In Eq. (9) there are two typical stationary solutions that are expressed analytically. The first is the in-phase state, $\hat{\phi}^{(0)}(x)=0$, and the second is $\hat{\phi}^{(0)}(x)=2 \pi x-\pi$ under periodic boundary conditions that are expressed as $\hat{\phi}(0, t)=$ $\hat{\phi}(2 \pi, t)+2 \pi$ and $\partial \hat{\phi}(x, t) /\left.\partial x\right|_{x=0}=\partial \hat{\phi}(x, t) /\left.\partial x\right|_{x=2 \pi}$. In this Appendix we perform a linear stability analysis of the in-phase solution. The corresponding analysis for $\hat{\phi}^{(0)}(x)=2 \pi x-\pi$ is performed in Appendix C. For the in-phase solution, Eq. (A1) becomes

$$
\frac{\partial \psi}{\partial t}=\left[k \frac{\partial^{2}}{\partial x^{2}}+A^{\prime}(0)\right] \psi
$$

The eigenfunctions $\psi_{n}$ and their corresponding eigenvalues $\lambda_{n}$ of the linear operator on the right-hand side of Eq. (A2) with the boundary conditions expressed in Eq. (4) are

$$
\begin{aligned}
\psi_{n}(x) & =\cos n \pi x, \\
\lambda_{n} & =-k(n \pi)^{2}+A^{\prime}(0) \quad(n=1,2, \ldots) .
\end{aligned}
$$

The results in Eq. (A3) show that when $k$ decreases from above, the in-phase state is destabilized at $k=k^{*}=A^{\prime}(0) / \pi^{2}$ together with the eigenmode $\cos \pi x$.

\section{APPENDIX B: REDUCTIVE PERTURBATION IN THE NEIGHBORHOOD OF $k=k^{*}$}

The results in Appendix A show that when $k<k^{*}$ the in-phase state becomes unstable. To investigate what type of stationary state arises after destabilization of the in-phase solution, we perform reductive perturbation in the neighborhood of the critical point $k=k^{*}[15]$.

Let us rewrite Eq. (9) by introducing a new parameter $\mu=k^{*}-k$ as

$$
\begin{aligned}
\frac{\partial \hat{\phi}(x, t)}{\partial t}= & k^{*} \frac{\partial^{2} \hat{\phi}(x, t)}{\partial x^{2}}+A^{\prime}(0) \hat{\phi}(x, t)+\left(-A^{\prime}(0) \hat{\phi}(x, t)\right. \\
& -\mu \frac{\partial^{2} \hat{\phi}(x, t)}{\partial x^{2}}+\int_{0}^{1} A\left(\hat{\phi}(x, t)-\hat{\phi}\left(x^{\prime}, t\right)\right) d x^{\prime} \\
& \left.-\int_{0}^{1} d x \int_{0}^{1} d x^{\prime} A\left(\hat{\phi}(x, t)-\hat{\phi}\left(x^{\prime}, t\right)\right)\right) .
\end{aligned}
$$

We then put a temporal parameter $\beta$ in front of the large brackets on the right-hand side of Eq. (B1) to distinguish the linearized terms with respect to $\hat{\phi}$ at $k=k^{*}$ from the remaining parts as

$$
\begin{aligned}
\frac{\partial \hat{\phi}(x, t)}{\partial t}= & k^{*} \frac{\partial^{2} \hat{\phi}(x, t)}{\partial x^{2}}+A^{\prime}(0) \hat{\phi}(x, t)+\beta\left(-A^{\prime}(0) \hat{\phi}(x, t)\right. \\
& -\mu \frac{\partial^{2} \hat{\phi}(x, t)}{\partial x^{2}}+\int_{0}^{1} A\left(\hat{\phi}(x, t)-\hat{\phi}\left(x^{\prime}, t\right)\right) d x^{\prime} \\
& \left.-\int_{0}^{1} d x \int_{0}^{1} d x^{\prime} A\left(\hat{\phi}(x, t)-\hat{\phi}\left(x^{\prime}, t\right)\right)\right) .
\end{aligned}
$$

When $\beta=1$, Eq. (B2) returns to Eq. (B1). We first investigate the case of $\beta=0$ in Eq. (B2):

$$
\frac{\partial \hat{\phi}(x, t)}{\partial t}=\left[k^{*} \frac{\partial^{2}}{\partial x^{2}}+A^{\prime}(0)\right] \hat{\phi}(x, t) .
$$

Expanding $\hat{\phi}$ in terms of the eigenfunctions of the linear operator on the right-hand side of Eq. (B3), which are given in Eq. (A3), as $\hat{\phi}(x, t)=\sum_{n=1}^{\infty} a_{n}(t) \psi_{n}(x)$, we have

$$
\frac{d a_{n}(t)}{d t}=\left(-n^{2}+1\right) A^{\prime}(0) a_{n}(t) \quad(n=1,2, \ldots) .
$$

Equation (B4) implies that for $\mu=0$ the modes with $n \geqslant 2$ in $\hat{\phi}$ are linearly stable, whereas the model with $n=1$ is neutral. That is, $a_{1}$ is constant in time. When $\beta \neq 0$ and $0<\mu \ll 1, a_{1}$ can change slowly with time. According to reductive perturbation theory (or, equivalently, to center manifold theory) $[15,21,22]$, the slow variable $a_{1}$ governs the asymptotic dynamics of the system, and the coefficients of the other modes, $a_{n}(n \geqslant 2)$, are expressed in terms of functions of $a_{1}$. Therefore, we formally write the dynamical equation that describes the asymptotic behavior of the system as

$$
\begin{aligned}
\frac{d a_{1}}{d t} & =G\left(a_{1}\right) \\
a_{n} & =g_{n}\left(a_{1}\right) \text { for } n \geqslant 2
\end{aligned}
$$

and we determine the forms of $G$ and $g_{n}$ by successive perturbative calculations using Eq. (B2). After some calculation, we obtain

$$
\begin{aligned}
& G=\mu \pi^{2} a_{1}+\left(\frac{A^{\prime \prime}(0)^{2}}{24 A^{\prime}(0)}+\frac{3}{8} A^{\prime \prime \prime}(0)\right) a_{1}^{3} \quad\left(\text { up to } a_{1}^{3}\right), \\
& g_{2}=\frac{a_{1}^{2} A^{\prime \prime}(0)}{12 A^{\prime}(0)} \quad\left(\text { up to } a_{1}^{2}\right), \\
& g_{n}=0 \quad\left(\text { up to } a_{1}^{2} \text { for } n \neq 2\right),
\end{aligned}
$$

where $\beta$ has returned to 1 . From Eq. (B5) we know that when $\frac{A^{\prime \prime}(0)^{2}}{24 A^{\prime}(0)}+\frac{3}{8} A^{\prime \prime \prime}(0)<0$ the system undergoes supercritical 
pitchfork bifurcation, whereas when $\frac{A^{\prime \prime}(0)^{2}}{24 A^{\prime}(0)}+\frac{3}{8} A^{\prime \prime \prime}(0)>0$ it undergoes subcritical bifurcation.

\section{APPENDIX C: SUPERCRITICAL HOPF BIFURCATION IN EQ. (9) UNDER PERIODIC BOUNDARY CONDITIONS}

As mentioned in Appendix A, if we apply periodic boundary conditions to Eq. (9), we obtain

$$
\hat{\phi}^{(0)}(x)=2 \pi x-\pi
$$

as a stationary solution of Eq. (9). This solution expresses a traveling wave in the original oscillation system, because $\phi(x, t)=(2 \pi x-\pi)+a t$, where $a$ is a constant given by $a=\int_{0}^{1} d x \int_{0}^{1} d x^{\prime} A\left(2 \pi\left(x-x^{\prime}\right)\right)$ obtained from Eq. (8). In this Appendix we examine the stability of the stationary solution, Eq. (C1), and show that the system undergoes a supercritical Hopf bifurcation at the value of $k$ where this solution becomes unstable. Let us start by finding the eigenfunctions $\varphi_{n}$ and their corresponding eigenvalues $\lambda_{n}$ of the linear operator $\int_{0}^{1} d x^{\prime} L\left(x, x^{\prime}\right)$ defined in Eq. (A1) with the stationary solution Eq. (C1). When $\varphi_{n}$ and $\lambda_{n}$ are found they can analytically be expressed as

$$
\begin{gathered}
\varphi_{n}(x)=e^{i 2 \pi n x}, \\
\lambda_{n}=-k(2 \pi n)^{2}-\frac{n}{2}\left(e^{i \alpha} \delta_{1 n}-e^{-i \alpha} \delta_{-1 n}+r \delta_{2 n}-r \delta_{-2 n}\right),
\end{gathered}
$$

where $\delta_{i j}$ is the Kronecker delta and $n$ takes all integer values except for 0 . This result indicates that when $\pi / 2<$ $\alpha$ there is a characteristic value of $k, k_{2}^{*}=-\cos \alpha / 8 \pi^{2}$, below which the stationary solution Eq. (C1) is unstable. At $k=k_{2}^{*}$ the eigenfunctions $\varphi_{1}$ and $\varphi_{-1}$ have purely imaginary eigenvalues $\lambda_{1}=-i \sin \alpha / 2$ and $\lambda_{-1}=i \sin \alpha / 2$, respectively, and the other modes have eigenvalues with negative real parts. This fact implies that if we expand $\hat{\phi}$ as $\hat{\phi}(x, t)=\sum_{n=-\infty, n \neq 0}^{\infty} a_{n}(t) \varphi_{n}(x)$ and consider the dynamics of the system described by Eq. (9) in the vicinity of $k=k_{2}^{*}$, the asymptotic behavior of the system can be described only by $a_{1}$ and $a_{-1}$. We note that $a_{1}$ and $a_{-1}$ are complex conjugates of each other. According to center manifold theory [15,21] we can determine the dynamic equations for $a_{1}$ and $a_{-1}$ and the form of $\hat{\phi}$. The results are as follows. Using real variables $A$ and $\theta$, introduced such that $A e^{i \theta}:=a_{-1}$, we can express $\hat{\phi}(x, t)$ as

$$
\hat{\phi}(x, t)=\hat{\phi}^{(0)}(x)+\left(A e^{i(\theta-2 \pi x)}+(\text { c.c. })\right)
$$

up to first order in $A$. The dynamic equations for $A$ and $\theta$ are given by

$$
\begin{aligned}
\frac{d A}{d t}= & 4 \mu_{2} \pi^{2} A \\
& +\frac{\left(24 r^{2}+19\right) \cos \alpha-26 r \cos 2 \alpha+5 \cos 3 \alpha-34 r}{4\left(-8 r \cos \alpha+3 \cos 2 \alpha+2 r^{2}+5\right)} A^{3},
\end{aligned}
$$$$
\frac{d \theta}{d t}=\omega_{0}-\frac{\left(-3 r \cos \alpha+\cos 2 \alpha+3 r^{2}+2\right) \sin \alpha}{-8 r \cos \alpha+3 \cos 2 \alpha+2 r^{2}+5} A^{2}
$$

up to the leading terms, where $\mu_{2}=k_{2}^{*}-k$ and $\omega_{0}=\sin \alpha / 2$. Since the coefficient of $A^{3}$ on the right-hand side of Eq. (C5) is negative in the parameter regions with which we are concerned, $\frac{\pi}{2}<\alpha<\pi$ and $0<r$, we find that the system undergoes supercritical Hopf bifurcation at $k=k_{2}^{*}$.

\section{APPENDIX D: PROPERTIES OF TWO-CLUSTER STATES EMERGING IN GLOBALLY COUPLED OSCILLATORS}

As previous work has shown $[6,11,13]$, when the connection function $A$, which represents the global interaction between oscillatory elements, involves higher harmonics, the system with $A$ can exhibit multicluster states where the oscillators form groups whose members have the same phase. In this Appendix, we focus on the main properties and linear stability analysis of the two-cluster state. Let us first denote the phases of the two clusters $\Phi_{1}$ and $\Phi_{2}\left(\Phi_{1}>\Phi_{2}\right)$, respectively, and denote the phase difference between the clusters

$$
\Delta=\Phi_{1}-\Phi_{2} .
$$

Next we denote the ratio of the number of oscillations $N_{1}$ belonging to cluster $\Phi_{1}$ to the total number of oscillations $N$ by

$$
p=N_{1} / N \text {. }
$$

The fraction of oscillations belonging to $\Phi_{2}$ is accordingly given by $1-p$. For the two-cluster state to be stationary, the parameters $p$ and $\Delta$ have to satisfy the following relation:

$$
p=\frac{A(0)-A(\Delta)}{2 A(0)-A(\Delta)-A(-\Delta)} .
$$

Linear stability analysis of the two-cluster state shows that the stability of the system is determined by the following three eigenvalues:

$$
\begin{aligned}
& \lambda_{1}=A^{\prime}(0) p+A^{\prime}(\Delta)(1-p), \\
& \lambda_{2}=A^{\prime}(-\Delta) p+A^{\prime}(0)(1-p), \\
& \lambda_{3}=A^{\prime}(\Delta)(1-p)+A^{\prime}(-\Delta) p .
\end{aligned}
$$

When $\lambda_{1}>0$ the cluster with phase $\Phi_{1}$ is unstable, whereas when $\lambda_{2}>0$ the cluster with phase $\Phi_{2}$ is unstable. For $\lambda_{3}>0$ the phase gap between the two clusters becomes unstable. The two-cluster state under consideration is only stable when

$$
\lambda_{i}<0 \text { for all } i=1,2,3 .
$$

In general, there are infinitely many two-cluster states that satisfy Eqs. (D3) and (D5) for a given $A$; i.e., the values of $p$ and $\Delta$ are not uniquely determined from only Eqs. (D3) and (D5).

\section{APPENDIX E: UNIQUE SELECTION OF THE VALUES OF $p$ AND $\Delta$ OF THE SYSTEM DUE TO THE LOCAL COUPLING TERM IN EQ. (9)}

For $k=0$ and $\alpha_{3}<\alpha<\alpha_{4}$ in Eq. (9), the system has twocluster states as its stationary state. As shown in Appendix D, there are infinitely many possible two-cluster states that satisfy the stable and stationary conditions given by Eqs. (D3) and (D5). In other words, the values of $p$ and $\Delta$ defined in Eqs. (D1) 
and (D2), which specify the two-cluster state, are not uniquely determined from only the stable stationary conditions [11]. However, our numerical simulations assert that if the system contains a local coupling term as in Eq. (9), the values of $p$ and $\Delta$ are uniquely determined. We prove this statement from an analytical point of view by restricting ourselves to the case where $0<k \ll 1$. We first note that in the stationary state, $\hat{\phi}(x)$ in Eq. (9) satisfies

$$
\begin{aligned}
k \frac{\partial^{2} \hat{\phi}}{\partial x^{2}}= & -\int_{0}^{1} A\left(\hat{\phi}(x)-\hat{\phi}\left(x^{\prime}\right)\right) d x^{\prime} \\
& +\int_{0}^{1} d x \int_{0}^{1} d x^{\prime} A\left(\hat{\phi}(x)-\hat{\phi}\left(x^{\prime}\right)\right) .
\end{aligned}
$$

We then expect that when $0<k \ll 1$, the $\hat{\phi}$ that satisfies Eq. (E1) is very close to a stationary solution of Eq. (9) for $k=0$. The latter is written as

$$
\hat{\phi}^{\text {test }}(x)=\Phi_{2} \Theta((1-p)-x)+\Phi_{1} \Theta(x-(1-p)),
$$

where $\Theta(x)$ is the Heaviside function defined in Eq. (12). $\Phi_{1}$ and $\Phi_{2}$ are constants that satisfy the relations Eq. (D1) and $p \Phi_{1}+(1-p) \Phi_{2}=0$, the latter coming from Eq. (7). These relations allow $\Phi_{1}$ and $\Phi_{2}$ to be expressed as

$$
\begin{aligned}
& \Phi_{1}=(1-p) \Delta, \\
& \Phi_{2}=-p \Delta .
\end{aligned}
$$

Since $p$ is related to $\Delta$ through Eq. (D3), and $\Phi_{1}$ and $\Phi_{2}$ are expressed as in Eq. (E3), the only parameter to be determined is $\Delta$. When $0<k \ll 1, \hat{\phi}$ and $\hat{\phi}^{\text {test }}$ may be different only near the interface between two plateau regions (clusters). Since the the width of the interface of $\hat{\phi}$ is of the order of $\sqrt{k}$ (see Fig. 4), the right-hand side of Eq. (E1) can approximately be written as

$$
\begin{aligned}
- & \int_{0}^{1} A\left(\hat{\phi}(x)-\hat{\phi}^{\mathrm{test}}\left(x^{\prime}\right)\right) d x^{\prime}+\int_{0}^{1} d x \int_{0}^{1} d x^{\prime} A\left(\hat{\phi}^{\mathrm{test}}(x)\right. \\
& \left.-\hat{\phi}^{\mathrm{test}}\left(x^{\prime}\right)\right) \\
= & -\left[A\left(\hat{\phi}(x)-\Phi_{1}\right) p+A\left(\hat{\phi}(x)-\Phi_{2}\right)(1-p)\right. \\
& -\left(\left(A(0) p+A\left(\Phi_{2}-\Phi_{1}\right)(1-p)\right) p\right. \\
& \left.\left.+\left(A\left(\Phi_{1}-\Phi_{2}\right) p+A(0)(1-p)\right)(1-p)\right)\right] \\
= & :-f(\hat{\phi}(x), \Delta) .
\end{aligned}
$$

Here, $f$ is a function of $\hat{\phi}$ and $\Delta$, defined above. If we introduce a quantity $V$ such that $V(\phi, \Delta)=\int_{0}^{\phi} f\left(\phi^{\prime}, \Delta\right) d \phi^{\prime}$, Eq. (E1) can be written as

$$
k \frac{\partial^{2} \hat{\phi}}{\partial x^{2}}=-\frac{\partial V}{\partial \hat{\phi}} .
$$

This equation is equivalent to the equation of motion of a particle moving in a potential $V$, if we regard $\hat{\phi}$ as the position of the particle and $x$ as time in Eq. (E5). In order for $\hat{\phi}$ to satisfy the conditions $\hat{\phi}(0)=\Phi_{2}, \hat{\phi}(1)=\Phi_{1}$, and $\hat{\phi}^{\prime}(0)=\hat{\phi}^{\prime}(1)=0$, $V$ must satisfy

$$
\begin{aligned}
& V\left(\Phi_{1}, \Delta\right)=V\left(\Phi_{2}, \Delta\right), \\
& \left.\frac{\partial V(\hat{\phi}, \Delta)}{\partial \hat{\phi}}\right|_{\hat{\phi}=\Phi_{1}, \Phi_{2}}=0 .
\end{aligned}
$$

We note that Eq. (E7) always holds because Eq. (E2) is a stationary solution of Eq. (9) for $k=0$. Equation (E6) gives the condition that determines the unique value of $\Delta$.
[1] S. Ishiwata, Y. Shimamoto, and N. Fukuda, Prog. Biophys. Mol. Biol. 105, 187 (2011).

[2] K. Sato, M. Ohtaki, Y. Shimamoto, and S. Ishiwata, Prog. Biophys. Mol. Biol. 105, 199 (2011).

[3] K. Sato, Y. Kuramoto, M. Ohtaki, Y. Shimamoto, and S. Ishiwata, Phys. Rev. Lett. 111, 108104 (2013).

[4] A. T. Winfree, J. Theor. Biol. 16, 15 (1967).

[5] Y. Kuramoto, Chemical Oscillations, Waves and Turbulence (Springer, New York, 1984).

[6] K. Okuda, Physica D 63, 424 (1993).

[7] A. C. Martin, M. Kaschube, and E. F. Wieschaus, Nature 457, 495 (2009).

[8] R. Kobayashi, A. Tero, and T. Nakagaki, J. Math. Biol. 53, 273 (2006).

[9] D. Battogtokh and A. Mikhailov, Physica D 90, 84 (1996).

[10] Y. Kobayashi and H. Kori, New J. Phys. 11, 033018 (2009).

[11] D. Hansel, G. Mato, and C. Meunier, Phys. Rev. E 48, 3470 (1993).

[12] P. Ashwin, O. Burylko, and Y. Maistrenko, Physica D 237, 454 (2008).
[13] H. Kori and Y. Kuramoto, Phys. Rev. E 63, 046214 (2001).

[14] I. Z. Kiss, C. G. Rusin, H. Kori, and J. L. Hudson, Science 316, 1886 (2007).

[15] H. Mori and Y. Kuramoto, Dissipative Structures and Chaos (Springer-Verlag, Berlin, 1998).

[16] S. H. Strogatz, Nonlinear Dynamics and Chaos: With Applications to Physics, Biology, Chemistry, and Engineering (Perseus Books, Cambridge, MA, 2000).

[17] C. A. Klausmeier, Theoret. Ecol. 1, 153 (2008).

[18] S. Ishiwata (private communication).

[19] S. K. Scott, Chemical Chaos (Oxford University Press, New York, 1993).

[20] P. Gaspard and X. J. Wang, J. Stat. Phys. 48, 151 (1987).

[21] J. Carr, Applications of Center Manifold Theory (SpringerVerlag, New York, 1981).

[22] S. Wiggins, Introduction to Applied Nonlinear Dynamical Systems and Chaos, 2nd ed. (Springer, New York, 2003).

[23] MATCONT is a MATLAB package for numerical bifurcation analysis of ODEs. A. Dhooge, W. Govaerts, and Yu. A. Kuznetsov, ACM Trans. Math. Softw. 29, 141 (2003). 